\title{
Bevacizumab plus IFN-alpha-2a in First-line Treatment of Patients With Advanced or Metastatic Renal Cell Carcinoma: A Prospective German Non-interventional Study
}

\author{
WOLFGANG SCHULTZE-SEEMANN ${ }^{1}$, HOLGER SCHULZ ${ }^{2}$, BARBARA TSCHECHNE $^{3}$ and MANUEL HÄCKL ${ }^{4}$ \\ ${ }^{1}$ University Hospital Freiburg (Albert-Ludwigs-University) Urology, Freiburg, Germany; \\ ${ }^{2}$ PIOH Study and Management GbR, Frechen, Germany; \\ ${ }^{3}$ Specialist for Internal Medicine Hematology and Oncology, Neustadt, Germany; \\ ${ }^{4}$ Roche Pharma AG Germany, Grenzach-Wyhlen, Germany
}

\begin{abstract}
Background/Aim: The combination of bevacizumab (BEV) plus interferon alpha-2a (IFN) constitutes an option for first-line treatment of metastatic renal cell carcinoma. Realworld data from routine clinical practice are rare and were, therefore, collected during this non-interventional study (NIS). Patients and Methods: A total of 359 patients received at least one dose of BEV (safety set population; SAF), 354 patients had at least one post-dose effectiveness assessment and formed the full analysis set (FAS) of the final analysis. Results: Progression-free survival (10.2 months, 95\% CI=8.6-12.6) and overall response rate (27.2\%) outcomes match the results from the phase III trials AVOREN and CALGB 90206. Longer overall survival (28.7 months, 95\% CI=24.5-38.3) probably is an effect of patient characteristics and follow-up therapies. Safety findings were comparable to the results of the Phase III trials, although comprehensive severity assessments were not provided. Conclusion: Overall, efficacy and safety data from $B E V$ plus IFN administered in routine clinical practice in an observational NIS are in line with results from the controlled phase III trials. (NCT02627144)
\end{abstract}

Renal cell carcinoma (RCC) has been predicted to occur in 9,900 men and 5,200 women in Germany for the year 2018, whereas in 2015, 3,306 deaths from renal carcinoma in men and 2,106 in women were documented by the German Robert Koch Institute (1). Renal malignancies can emerge

Correspondence to: Prof. Dr. Wolfgang Schulze-Seemann, University Hospital Freiburg, Clinic for Urology, Hugstetter Street 55, 79106 Freiburg. Tel: +49 76127028920, Fax: +49 76127028780, e-mail: wolfgang.schultze-seemann@uniklinik-freiburg.de; sekretariat. schultze-seemann@uniklinik-freiburg.de

Key Words: Renal cell carcinoma, advanced disease, metastatic disease, bevacizumab (Avastin ${ }^{\circledR}$ ), non-interventional study. from different tissues. With more than $90 \%$ of all renal malignancies, renal cell carcinoma represents the most frequent form; among these, about $80 \%$ are clear cell renal carcinomas histologically (2). Relative 5-year survival rate is $77 \%$ in male and female patients, as well (1). Median age at first diagnosis is 67 years in men and 72 years in women (1). Therefore, RCC is a malignancy of higher age and has a relatively positive prognosis. Most relevant risk factors are active (and passive) smoking, hypertension and overweight, lack of physical exercise and renal insufficiency from any reason (1). Exposition to chemical agents, such as lead, analgesics, or asbestos, has been linked to a higher incidence of RCCs $(3,4)$. Up to $75 \%$ of RCCs are diagnosed in organconfined stages (T1 and T2), whereas one fourth is already locally advanced or presenting as metastatic disease having a poor prognosis (1).

RCC is mainly refractory to classic chemotherapy regimen (5). Its biology is possibly influenced by the immune system and is considered an immune responsive disease (6). Therefore, immunotherapy with interferon-alpha, besides surgical intervention (removal of primary tumor lesion) had been the standard of care so far, until anti-vascular endothelial growth factor (VEGF) antibodies and other VEGF receptor (VEGFR)-targeted therapies (tyrosine kinase inhibitors; TKIs) were introduced to the treatment regimen (7-10). Mutation of the VHL tumor suppressor gene is observed in up to $80 \%$ in clear cell kidney cancers, leading to increased rates of VEGF secretion in RCC (11), thus being a promising candidate for VEGF-targeted therapy. First-line sunitinib (TKI) (9), the combination of BEV plus interferon (IFN)-alpha-2a (7), and temsirolimus (12), were shown to be better than monotherapy with IFN. First-line pazopanib, another TKI, has also been tested against placebo in RCC therapy (10). All these treatment options are approximately comparable in terms of progression-free survival (PFS) and can be used under the premise of favorable to moderate prognosis according to the 
Memorial Sloan-Kettering Cancer Center (MKSCC)-criteria for first-line therapy of advanced or metastatic RCC. In consequence, actual treatment guidelines (12) recommend a targeted first-line therapy with VEGFR-inhibitors, like sunitinib (9), pazopanib (10), or bevacizumab plus immune stimulative IFN, for patients with advanced or metastatic RCC with low to intermediate risk [per Motzer- or MSKCC-Score (13) /IDMC score International Metastatic Database Consortium (IMDC) adverse risk factors] (8).

Bevacizumab (BEV), a recombinant monoclonal antibody that selectively binds to the human VEGF was approved for treatment of advanced or metastatic RCC in 2008, based on the results of study BO17705 (8); that study demonstrated a doubling for mPFS and overall response rate (ORR) for patients in the experimental arm with BEV plus IFN compared to IFN plus placebo.

The intention of this non-interventional study was to prospectively document data on safety and effectiveness of $\mathrm{BEV}$ in combination with IFN as first-line treatment in patients with advanced or metastatic RCC, in routine clinical practice. Treatment approaches should follow the specifications of the actual Summary of Product Characteristics (SmPC) for BEV. Main parameters of interest were PFS, ORR, and adverse events including adverse events of special interest of the combination therapy. Other parameters of interest were treatment duration and reasons for treatment discontinuation, dosing of drug and treatment stops. Results reflect the use of $\mathrm{BEV}$ plus IFN in routine clinical practice of day to day practice in oncological and urological specialist centers, hospitals, and open care practices involved in the treatment of metastatic RCC (mRCC) patients, in Germany. BEV is recommended to be administered every 2 weeks at a dosage of $10 \mathrm{mg} / \mathrm{kg}$ bodyweight. Starting dose of IFN was 9 MIU three times a week. Data acquisition through this non-interventional study (NIS) deemed the best option to compare data from real world documentation with results of the clinical studies AVOREN (14) and CALGB $90206(15,16)$. New treatment options in mRCC emerge, for example anti-VEGF in combination with cancer immunotherapies (e.g., atezolizumab plus BEV, NCT02420821 or nivolumab plus BEV NCT02210117). The aim of investigating immune-checkpoint inhibitors in combination with anti-angiogenic agents like anti-VEGF antibodies or TKIs is to improve tumor outcome and tolerability of the treatment in patients with advanced or metastatic RCC. During the initial use of these combination therapies, it would be important to determine that how BEV is handled in routine clinical practice in $\mathrm{mRCC}$.

\section{Patients and Methods}

Estimated outcomes were based on the AVOREN-study results (14) and provided the basis for the calculation of the patient population for this NIS with 400 advanced RCC patients. Statistical assumptions included the estimation of the following treatment effects: ORR 30.6\% (95\% CI=26.1\%-35.1\%), median PFS 10.2 months (95\% CI 9.5-10.9) and 12-month PFS of $44.2 \%(95 \%$ $\mathrm{CI}=41.7 \%-6.6 \%$ ), including $99 \%$ probability of detection of less frequent adverse drug effects (occurring at a rate of 2.5\%) 4 times during this NIS

Between January 2008 and September 2014, 407 patients were identified at the 136 participating medical centers across Germany. Due to lack of valid informed consent, lack of documentation and other reasons identified by the data review committee, out of these 407 patients, 354 patients were analyzed in the full analysis set (FAS) of this NIS (Figure 1).

Adult patients were included in this prospective non-interventional observational study after histological confirmation of advanced or metastatic RCC. Exclusion of contraindications against the combination therapy and decision for treatment with BEV and IFN were made by the treating physician or the medical committee on the treatment routine. According to the SmPC of BEV, patients received first-line-treatment with BEV at a dose of $10 \mathrm{mg} / \mathrm{kg}$ body weight every two weeks in combination with IFN immunotherapy, after they consented to participation and data documentation within this NIS. Patients with hypersensitivity against the antibody or its constituents, CHO-cell-products, or other recombinant or humanized antibodies, or pregnant patients were not eligible for treatment with BEV. Additionally, patients with contraindications against immunotherapy with IFN were ineligible for treatment within this NIS. All eligible patients obtained their medication as normal merchandise. According to SmPC, treatment with BEV should be applied until progression of underlying renal carcinoma or no further clinical benefit.

Concomitant diseases and co-medications were documented at baseline. During treatment phase of BEV plus IFN administration, best tumor response over time was assessed as per clinical routine of the individual center, lab-values, staging assessments in case of suspected change in underlying malignant disease, and adverse events for the combination BEV plus IFN were captured by the study sites.

Ethical committee vote was obtained from the Ethics Committee of the Freiburg university hospital (scientific lead Prof. SchultzeSeemann) prior to start of the NIS.

A data review meeting prior to data base lock defined, besides the "all patients" set ( $\mathrm{AP}, \mathrm{N}=365$ ), the safety set ( $\mathrm{SAF}, \mathrm{N}=359$ patients having received at least one dose of BEV), the full analysis set (FAS, $\mathrm{N}=354$ patients having received at least one dose of BEV and at least one post dose effectiveness assessment) and the per protocol set (PP, $\mathrm{N}=353$ consisting of all patients of the FAS without major protocol deviations). All effectiveness parameters will be described for the FAS, all other parameters refer to the SAF (Figure 1).

The NIS ended in September 2016, covering a rather elongated study period of 81 months. The observational plan was amended several times, at last in November 2010 (version 2.2, Nov 18th2010).

During the conduct of the study, a change of the supporting Clinical Research Organization occurred. The results from this non-comparative observational study reflect the day to day practice and do not adhere to strict requirements of a study protocol, thereby reflecting and presenting real-world data, including all the relevant uncertainties.

Statistical analysis. The predefined statistical analysis plan was followed using SAS ${ }^{\circledR}$ package version 9.4. Quantitative data were analyzed descriptively depicting mean, standard deviation (SD), 
minimum and maximum values, as well as, median and interquartile range (IQR; Q1-Q3). Qualitative and categorical variables are shown as means of absolute and relative frequency distributions PFS and overall survival (OS) were estimated using Kaplan-Meierapproach and corresponding 95\% confidence intervals (CI). Percentages for relative frequencies were based on non-missing values $(100 \%)$. Adverse events were analyzed on patient basis (incidental rate for specific event). Adverse events and medical history were coded using MedDRA version 15.1.

\section{Results}

Baseline characteristics of the 359 patients from the SAF displayed a median age of 68.0 years $(\mathrm{IQR}=59.0-73.0)$ with $40.3 \%$ of patients younger than 65 years and $16.8 \%$ older than 75 years. Among the SAF, $68 \%$ were male patients, median bodyweight was $80.0 \mathrm{~kg}(\mathrm{IQR}=70.0-90.0)$ with a median BMI of $26.8 \mathrm{~kg} / \mathrm{m} 2$ (IQR=24.5-30.5). The majority $(87.2 \%)$ of this patient set histologically had a clear cell carcinoma, $3.2 \%$ a papillary, $1.4 \%$ a chromophobe and one patient $(0.5 \%)$ had a collecting duct carcinoma; entry was missing for 141 patients. At time of initial diagnosis of the mRCC, $9.3 \%$ of patients had stage I, $10.1 \%$ stage II, $21.5 \%$ stage III and $59.1 \%$ stage IV (however, data from about one third of the patients $(\mathrm{n}=122)$ was missing). A percentage of $91.0 \%$ have had surgery of their renal malignancy executed 12.0 months (IQR=2.0-47.0) before diagnosis at initiation of the study. At time of inclusion into this NIS, the tumor stage was locally advanced stage III in $28.1 \%$ and stage IV in $71.9 \%$. Metastases were present in lung $69.3 \%$, lymph nodes $26.4 \%$, bones $23.2 \%$, and brain $4 \%$. Most of the patients (76.9\%) had a medical history or co-morbidity, most frequently vascular disorders $54.6 \%$-mainly hypertension (54\%), 23.7\% cardiac disorders, $23.1 \%$ metabolism and nutrition disorders -mainly diabetes mellitus (17.3\%), $19.5 \%$ had renal and urinary disorders (19.2\% nephropathy) and $5.3 \%$ respiratory, thoracic, and mediastinal disorders -mostly chronic obstructive pulmonary disease (3.6\%). Additional baseline characteristics are displayed in Table I comparing the population of this NIS with two interventional phase III trials.

Median total observation period for SAF was 217.5 days ( $I Q R=106.0-415.0)$, with a median treatment duration with BEV of 192.0 days (IQR=85.0-394.0) calculated for patients with data available. The mean total number of cycles of systemic therapy was $16.6(\mathrm{SD}=14.0)$, with a minimum of 1 cycle up to a maximum of 71 cycles. Among SAF patients, $33.4 \%$ received 20 cycles of $\mathrm{BEV}$ or more. A total of $24.8 \%$ of the patient population still received treatment by the end of the first year of observation and $4.2 \%$ by the end of the second year. Mean dose of BEV per cycle was $10.6 \mathrm{mg} / \mathrm{kg}$ body weight $(\mathrm{BW})(\mathrm{SD}=7.0)$, matching the specifications of the SmPC. Interferon-alpha-2a was used throughout all treatment cycles as main combination partner at a median dose of 3.0 million IU.

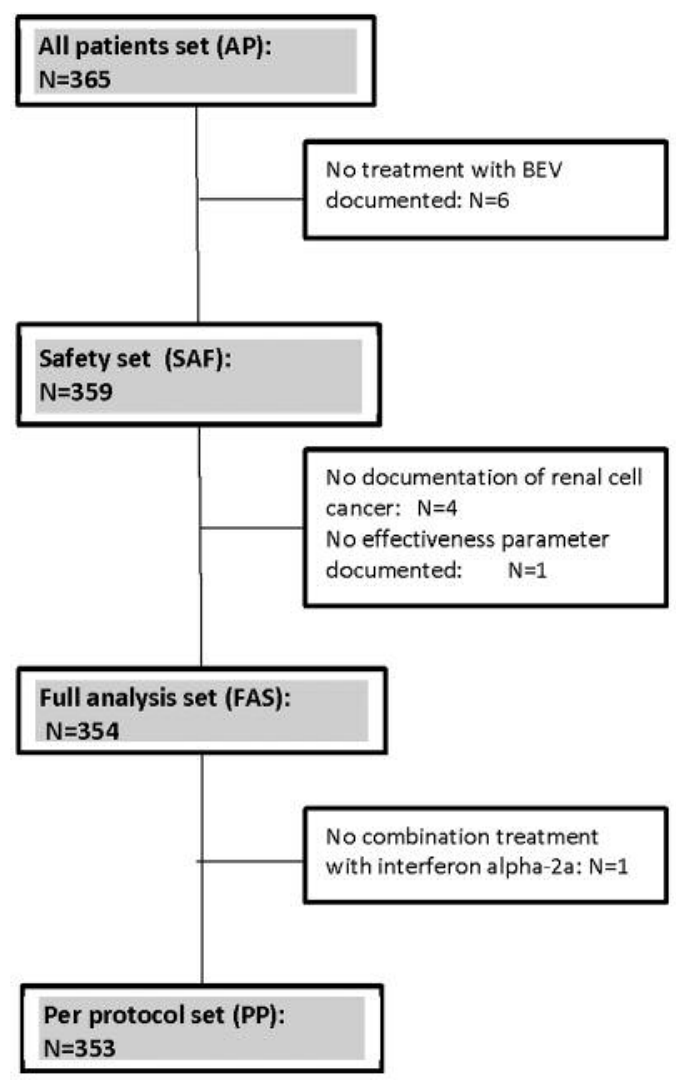

Figure 1. Consort diagram. BEV, Bevacizumab.

Progression of underlying malignant disease was the main reason for treatment discontinuation (51.8\%) during observation period within the SAF. About $25 \%$ of patients stopped medication due to administrative reasons (not described further), while $12.0 \%$ of participants refused to continue treatment or indicated poor compliance. Thirty-five patients $(10.2 \%)$ died from the underlying malignancy and treatment was stopped due to serious adverse events in 32 patients $(9.4 \%)$. After 52 weeks (end of observation) more than half of the SAF $(54.0 \%)$ were planned to receive subsequent therapies. These data correspond with the followup information on second-line therapies, collected 12 months after the observation period, with $45.4 \%$ of safety population having in fact been treated with subsequent therapies. Sorafenib was administered as $2 \mathrm{~L}$-therapy to $5.8 \%$ of the patients, another $30.6 \%$ received antineoplastic agents with various substances, and $11.4 \%$ received immunosuppressive everolimus. Immunostimulants, like interferon, were prescribed in $7(1.9 \%)$ patients and $2.5 \%$ received BEV as follow-up treatment.

Median PFS was 10.2 months (95\% CI=8.6-12.6) for the FAS $(\mathrm{N}=354)$, with $50 \%$ of patients attaining a PFS between 
Table I. Patient baseline characteristics (NIS, AVOREN, CALGB 90206).

\begin{tabular}{|c|c|c|c|c|c|c|c|c|c|c|}
\hline & \multirow{2}{*}{\multicolumn{2}{|c|}{$\begin{array}{l}\text { NIS AVA RCC } \\
\text { BEV plus IFN } \\
\text { (SAF) N=359 }\end{array}$}} & \multicolumn{4}{|c|}{ AVOREN (8) } & \multicolumn{4}{|c|}{ CALGB 90206 (17) } \\
\hline & & & \multicolumn{2}{|c|}{$\begin{array}{c}\text { BEV plus IFN } \\
\mathrm{N}=327\end{array}$} & \multicolumn{2}{|c|}{$\begin{array}{l}\text { IFN plus placebo } \\
\quad \mathrm{N}=322\end{array}$} & \multicolumn{2}{|c|}{$\begin{array}{c}\text { BEV plus IFN } \\
\mathrm{N}=369\end{array}$} & \multicolumn{2}{|c|}{$\begin{array}{l}\text { IFN plus placebo } \\
\qquad \mathrm{N}=363\end{array}$} \\
\hline & $\mathrm{n}$ & $\% * /$ range & $\mathrm{n}$ & $\% /$ range & $\mathrm{n}$ & $\% /$ range & $\mathrm{n}$ & $\% /$ range & $\mathrm{n}$ & $\% /$ range \\
\hline Age (median) & 68 & $59-73$ & 61 & $30-82$ & 60 & $18-81$ & 61 & $56-70$ & 62 & $55-70$ \\
\hline Male & 242 & 68 & 222 & 68 & 234 & 73 & 269 & 73 & 239 & 66 \\
\hline Female & 114 & 32 & 105 & 32 & 88 & 27 & 100 & 27 & 124 & 34 \\
\hline Karnofsky score 90-100/ECOG 0-1 & 187 & 55 & & 76 & & 78 & & 98 & & 99 \\
\hline \multicolumn{11}{|l|}{ Risk score } \\
\hline Favorable & 114 & 36 & 87 & 27 & 93 & 29 & 97 & 26 & 95 & 26 \\
\hline Intermediate & 159 & 50 & 183 & 56 & 180 & 56 & 234 & 64 & 231 & 64 \\
\hline Poor & 21 & 6 & 29 & 9 & 25 & 8 & 38 & 10 & 37 & 10 \\
\hline N.a. & 22 & 6 & 28 & 9 & 24 & 7 & & & & \\
\hline \multicolumn{11}{|l|}{ Metastatic sites } \\
\hline Lung & 242 & 69 & 192 & 62 & 179 & 59 & 252 & 68 & 254 & 70 \\
\hline Lymph node & 92 & 26 & 107 & 34 & 107 & 36 & 130 & 35 & 129 & 36 \\
\hline Liver & & & 57 & 18 & 56 & 19 & 74 & 20 & 73 & 20 \\
\hline Bone & 81 & 23 & 58 & 18 & 65 & 20 & 104 & 28 & 109 & 30 \\
\hline Brain & 14 & 4 & n.r. & & n.r. & & n.r. & & n.r. & \\
\hline Previous nephrectomy & 323 & 91 & & 100 & & 100 & 312 & 85 & 308 & 85 \\
\hline
\end{tabular}

* No display of decimal places in order to align with data from AVOREN and CALGB manuscripts. NIS, Non-interventional study; AVA, Avastin ${ }^{\circledR}$ (bevacizumab); BEV, bevacizumab; IFN, interferon alpha-2a; SAF, safety set; ECOG, eastern co-operative oncology group performance status. N.a., not available; n.r., not reported.

Table II. Effectiveness outcomes (NIS, AVOREN, CALGB 90206).

\begin{tabular}{|c|c|c|c|c|c|c|c|c|c|c|}
\hline & \multirow{2}{*}{\multicolumn{2}{|c|}{$\begin{array}{l}\text { NIS AVA RCC } \\
\text { BEV plus IFN } \\
\text { (FAS) N=354 }\end{array}$}} & \multicolumn{4}{|c|}{ AVOREN (14) } & \multicolumn{4}{|c|}{ CALGB 90206 (15) } \\
\hline & & & \multicolumn{2}{|c|}{$\begin{array}{c}\text { BEV plus IFN } \\
\mathrm{N}=327\end{array}$} & \multicolumn{2}{|c|}{$\begin{array}{l}\text { IFN plus placebo } \\
\quad \mathrm{N}=322\end{array}$} & \multicolumn{2}{|c|}{$\begin{array}{c}\text { BEV plus IFN } \\
\mathrm{N}=369\end{array}$} & \multicolumn{2}{|c|}{$\begin{array}{l}\text { IFN plus placebo } \\
\qquad N=363\end{array}$} \\
\hline & median & $95 \% \mathrm{CI}$ & $\mathrm{n}$ & $95 \% \mathrm{CI}$ & $\mathrm{n}$ & $95 \% \mathrm{CI}$ & $\mathrm{n}$ & $95 \% \mathrm{CI}$ & $\mathrm{n}$ & $95 \% \mathrm{CI}$ \\
\hline PFS (months) & 10.2 & $8.6-12.6$ & 10.2 & $7.5-9.7$ & 5.4 & $3.1-5.6$ & 8.5 & $7.5-9.7$ & 5.2 & $3.1-5.6$ \\
\hline OS (months) & 28.7 & $24.5-38.3$ & 23.3 & & 21.3 & & 18.3 & $16.5-22.5$ & 17.4 & $14.4-20.0$ \\
\hline ORR $(\%)$ & 27.2 & & 31.4 & & 12.8 & & 25.5 & $20.9-30.6$ & 13.1 & $9.5-17.3$ \\
\hline \multirow[t]{2}{*}{ Duration of BEV- treatment } & 13.0 cycles/ & (range) & 9.7 & (range) & 5.1 & (range) & 6 & (range) & 3 & (range) \\
\hline & 6.5 months & $1-71$ & months & $0-24.4$ & months & $0-24.0$ & cycles & $1-38$ & & $1-36$ \\
\hline
\end{tabular}

NIS, Non-interventional study; AVA, Avastin ${ }^{\circledR}$ (bevacizumab); BEV, bevacizumab; IFN, interferon alpha-2a; FAS, full analysis set; CI, confidence interval; PFS, progression free survival; OS, overall survival; ORR, overall response rate; RCC, renal cell carcinoma; CALGB, Cancer and Leukemia Group B.

4.2 and 18.5 months. Tumor response was assessed according to clinical routine of day-to-day practice by the individual participating centers. Eighteen patients $(5.3 \%)$ of the FAS were documented with a complete response (CR), 74 patients $(21.9 \%)$ showed a partial response (PR), and most of the patients $(39.1 \%)$ had stable disease according to the investigator assessment. Progression of disease occurred in 56 patients $(16.6 \%)$, while 58 patients $(17.2 \%)$ were not evaluable. ORR resulted in $27.2 \%$ of the FAS and disease control rate (DCR) was $66.3 \%$. Of note, $67.1 \%$ of the FAS patients had a Karnofsky index of $80-100 \%$ or a mean Karnofsky index of $78.3 \% \quad(\mathrm{SD}=16.5)$ by the end of observation, with a mean Karnofsky index of $85.7 \%$ $(\mathrm{SD}=11.7)$ at the beginning of the study or $55 \%$ of the patient ranging within Karnofsky indices between 90-100\%. Median OS was 28.7 months (95\% CI=24.5-38.3). KaplanMeier-based 12-month OS rate was 76\% (95\% CI=71-80\%) for the FAS. 
Safety assessments within this observational study included documentation of adverse events and laboratory abnormalities of BEV in combination with IFN. In order to maximize adherence to safety reporting, case record form (CRF) documentation asked for adverse event occurrence during the actual observation period, and if so, appropriate specification was required at each visit. In contrast to the underreporting of adverse events in many other NIS, altogether 11,377 adverse events for the combination therapy were reported in 334 patients (93\%) of the SAF. However, it needs to be clarified that this figure depicts the elementary events, not episodes or incidences. Serious adverse events (SAE) were seen in 72 patients $(20.1 \%)$, whereas 70 patients (19.5\%) of the SAF were documented with an AE of special interest (AESI) such as respiratory, vascular, gastrointestinal, nervous system, renal, and urinary or general disorders. Blood and lymphatic system disorders were the most frequently reported system organ class (SOC) by MedDRA in 252 patients $(70.2 \%)$, with anemia occurring in 198 patients $(55.2 \%)$, leukopenia in 150 patients $(41.8 \%)$ and thrombocytopenia in 118 patients $(32.9 \%)$. Toxicities $\geq$ grade 3 within this SOC were observed in 47 patients $(12.1 \%)$, anemia was seen in $28(7.8 \%)$ patients and neutropenia grade 3 in $10(2.8 \%)$. More than half of patients $(n=186)$ reported gastrointestinal disorders (SOC gastrointestinal disorders). Nausea $(39.3 \%)$ and diarrhea $(22.3 \%)$ were the predominant events. Proteinuria as a renal symptom was documented in 90 patients $(25.1 \%)-7$ patients $(1.9 \%)$ had grade 3 proteinuria, 33 patients had cardiac disorders, 4 had cardiac failure grade 3 . Among AESIs, epistaxis was the most frequently reported $(9.7 \% ; 35$ patients); one episode of grade 3 epistaxis. Hemorrhages (one gastrointestinal, one cerebral and one post procedural) $\geq$ grade 3 occurred in 3 patients.

A total of $143(40.9 \%)$ patients out of the SAF were reported to have died during the observational and follow-up documentation of this NIS, 120 patients due to progression of their underlying disease. Seventeen of the remaining 23 patients died from various reasons (4 from cardiac decompensation, 3 from pneumonia and cardiac failure, 2 from GI-perforation, 8 from different other reasons) and for 6 patients the cause of death was not documented.

\section{Discussion}

For a long period of time, IFN has been the standard of care in first-line therapy for mRCC. This regimen allowed for an ORR of $10-15 \%$ and a mean OS of about 12 months. (17). The pivotal European AVOREN study (NCT00738530) (8, 14) and the US American phase III CALGB trial (NCT00072046) $(15,16)$ established BEV plus IFN in firstline therapy, as both randomized controlled trials (RCTs) confirmed the superiority of the combination of BEV plus IFN over IFN monotherapy.
Median PFS and ORR, calculated as percentage of patients with $\mathrm{CR}$ and $\mathrm{PR}$, were defined as effectiveness parameters of primary interest in this NIS, besides the monitoring of safety. Effectiveness and safety data from this observational study were compared to the phase III trials, under proviso of the totally different approaches of data acquisition within controlled studies and the merely observational data documentation in this NIS.

Patient baseline characteristics from the three different studies are listed in Table I. Patients treated within the current NIS were older and the percentage of patients with a nonimpaired Karnofsky index (90-100\%) at baseline was lower compared to the clinical trial populations. These are -expected findings for a real world (RW) population (Table I).

Comparison of the median duration of BEV plus IFN treatment in the pivotal AVOREN study (9.7 months) with that observed in the current study (6.5 months or 13 cycles) reflects a shorter than expected treatment duration in routine practice. However, direct comparison to the CALGB trial (8.2 cycles), demonstrated a longer median duration of treatment in the present study.

Outcomes for PFS of the actual observational study completely matched the findings from AVOREN and CALGB (Table II).

ORR $(27.2 \%)$ reported from the day to day practice for the combination of BEV plus IFN, with no strict parameters for assessment of response, ranged between results from CALGB (25.5\%) and AVOREN (31.4\%) study (Table II). Eighteen patients $(5.3 \%)$ were documented with CR in this non-interventional observation, while only 4 patients $(1 \%)$ in AVOREN. However, it should be considered that in the RCT, only patients with a measurable disease at baseline were included into the response analysis.

Median OS was 28.7 months; longer compared to the AVOREN (14) or CALGB-results (15). Nevertheless, it should be noted that patients on average were older in the NIS, but probably with a slightly lower risk score at baseline. It should also be discussed whether OS-effects were confounded by the recent subsequent treatment(s) that patients in this NIS received. Since years, the best sequence of therapies (achieving the best OS results) including novel therapeutic approaches have been extensively discussed and reviewed $(18,19)$. Identification of patients likely to benefit from one or another treatment regimen, including a possible role of biomarkers for selection and response prediction, or models with risk factors for treatment related toxicities (20) have been considered in this context. The final analysis of the CALGB-study described a survival difference according to a patient's risk score at baseline. A tremendous difference existed between the OS outcomes for good-risk patients of 32.5 months for BEV plus IFN compared to 33.5 for IFN, 17.7 months for BEV plus IFN and 16.1 months for IFN for patients with intermediate risk and for high-risk patients 
6.6 months in BEV plus IFN vs. 5.6 months in IFN. Subgroup analyses had not been pre-specified for the study and calculation on patient numbers were not powered to show any statistical significance between the risk-adapted subgroups (14). As the patient cohort of this NIS tended to enroll more patients with a favorable risk score $(36 \%$ vs. $27 \%$ and $26 \%$ in AVOREN and CALGB, respectively) this could be one explanation for the observed longer OS outcome.

Compared to both RCTs, nature and quality of adverse events within this observational study were matchable. Presentation of safety data from AVOREN, CALGB and the actual NIS, however, differed methodologically. In consequence, a quantitative comparison between the RCTs and these observational data was not possible. Summarizing the outcomes, frequently reported adverse events from AVOREN and CALGB, like fatigue or proteinuria, were documented at a low rate in the present study, probably due to a tendency towards underreporting of anticipated effects. However, anemia, which might also be related in part to the underlying malignant disease, and thrombocytopenia were reported rather frequently in this NIS. AESIs having been observed in clinical trials with BEV, such as hemorrhages, gastrointestinal perforations, fistula, arterial and venous thrombotic events, and impaired wound healing were reported with a low incidence.

The overall safety profile from this unselected patient population did not differ from the results of the randomized trials, though the non-interventional character of the underlying observation with all its possible limitations must be considered. Based on the reported data, the safety profile of the combination of BEV plus IFN was consistent with the known safety risks.

RCC has been categorized as an immune-responsive disease since first studies with immunostimulants showed positive effects. At present, immune checkpoint inhibitors, amongst others, are in development for the treatment of mRCC. Programmed cell death 1 (PD-1) is an inhibitory immune checkpoint, and blockade of the PD-1 cascade is an interesting target in mRCC, aiming at restoration of anti-cancer immunity. Expression of the ligand for PD-1 in $\mathrm{mRCC}$ has been shown to be a negative prognostic factor. Promising results have been observed in several settings with nivolumab an anti-PD-1inhibitor in RCC $(21,22)$, while other immune checkpointinhibitors are currently being evaluated for therapeutic use in mRCC. The results from a phase III-study (CheckMate 214) comparing the anti-PD-1 inhibitor nivolumab in combination with the anti-cytotoxic T-lymphocyte antigen-4 (CTLA-4) antibody ipilimumab versus sunitinib showed a statistically significant OS prolongation, significantly improved ORR and a prolonged median PFS for mRCC patients (23). A phase IB/II study evaluating lenvatinib (an oral multikinaseinhibitor) and pembrolizumab (an anti-PD-1 monoclonal antibody) showed first positive results in terms of ORR, duration of response (DOR), and PFS in treatment-naïve and pre-treated patients, as well as in PD-L1-positive or PD-L1negative mRCC patients (24). Treatment options based on the established principle of VEGF-inhibition plus immunotherapy are tested in mRCC to optimize effectiveness, safety, and quality of life for affected patients $(25,26)$. Actual results from a phase II-study (IMmotion150) investigating the effect of the anti-PD-L1 atezolizumab in combination with the antiVEGF bevacizumab showed encouraging results when compared to sunitinib in terms of PFS $(26,27)$. A Phase-III study (IMmotion151) confirmed the clinical benefit of this combination therapy versus sunitinib in terms of improved PFS in patients with PD-L1-positive disease. While OS results are still immature, albeit being promising, patients treated with atezolizumab plus bevacizumab showed a delayed interference of symptoms with daily life and a less frequent occurrence of grade 3 or 4 events when compared to sunitinib (24). It will be a matter of future discussion and analyses (28) to establish an optimal sequencing of available therapies for all the specific patient subgroups in advanced or metastatic RCC, and moreover to ultimately evaluate efficacy and tolerability of the combinations between targeted and immune-based therapies (anti-PD1, anti-PDL-1, CTLA-4, dendritic cell vaccines, anti VEGF), which are subjects of effective clinical investigations.

\section{Conclusion}

Targeted therapy with BEV plus IFN is still recommended as first-line therapy for patients with advanced or metastatic RCC and good to intermediate risk $(29,30,31)$. The predefined statistical assumptions of this NIS were approximated by the study outcomes, except a somewhat lower than expected ORR (30.6vs. 27.2\%). The overall safety profile of this day-to-day clinical practice observation was comparable to findings from the two randomized trials. On cautionary note, all data presented with this report may be subject to limitations, based on the less regulated administration, documentation, and patient selection during data collection in a NIS. However, results demonstrate that outside the strict limitations and regulations -including inclusion and exclusion parametersimposed by a controlled clinical trial, the combination of BEV plus IFN works effectively in RCC patient treatment. Observational data from clinical routine, as well as data generated from AVOREN or CALGB 90206 trials, showed preponderant consistency. Moreover, results from this observational study offer perspectives towards new combinations of BEV with modern cancer immunotherapies.

\section{Acknowledgements}

The Authors thank all the patients, caregivers, and investigators who participated in this non-interventional study. Study report and statistical analyses were provided by AMS, Advanced Medical Services GmbH, Mannheim. They also thank Dorothee Schneider, S\&P GmbH, who provided medical writing support. 


\section{References}

1 Robert Koch Institut: Krebs in Deutschland. Nierenkrebs, 2017.

2 Ljungberg B, Bensalah K, Canfield S, Dabestani S, Hofmann F, Hora M, Kuczyk MA, Lam T, Marconi L, Merseburger AS, Mulders P, Powles T, Staehler M, Volpe A and Bex A: EAU guidelines on renal cell carcinoma: 2014 update. Eur Urol 67(5): 913-924, 2015.

3 Boffetta P, Fontana L, Stewart P, Zaridze D, SzeszeniaDabrowska N, Janout V, Bencko V, Foretova L, Jinga V, Matveev V, Kollarova H, Ferro G, Chow WH, Rothman N, van Bemmel D, Karami S, Brennan P and Moore LE: Occupational exposure to arsenic, cadmium, chromium, lead and nickel, and renal cell carcinoma: a case-control study from Central and Eastern Europe. Occup Environ Med 68(10): 723-728, 2011.

4 Kabaria R, KLaassen Z and Terris MK: Renal cell carcinoma: links and risks. Int J Nephrol Renovasc Dis 9: 45-52, 2016.

5 Motzer R J and Russo P: Systemic therapy for renal cell carcinoma. J Urol 163(2): 408-417, 2000.

6 Zisman A, Pantuck AJ and Belldegrun A: Immune and genetic therapies for advanced renal cell carcinoma. Rev Urol 2(1): 5460, 2000.

7 Motzer RJ, Bacik J, Murphy BA, Russo P and Mazumdar M: Interferon-alfa as a comparative treatment for clinical trials of new therapies against advanced renal cell carcinoma. J Clin Oncol 20(1): 289-296, 2002.

8 Escudier B, Pluzanska A, Koralewski P, Ravaud A, Bracarda S, Szczylik C, Chevreau C, Filipek M, Melichar B, Bajetta E, Gorbunova V, Bay JO, Bodrogi I, Jagiello-Gruszfeld A and Moore N; AVOREN Trial investigators: Bevacizumab plus interferon alfa-2a for treatment of metastatic renal cell carcinoma: a randomised, double-blind phase III trial. Lancet 370(9605): 2103-2111, 2007.

9 Motzer RJ, Hutson TE, Tomczak P, Michaelson MD, Bukowski RM, Rixe O, Oudard S, Negrier S, Szczylik C, Kim ST, Chen I, Bycott PW, Baum CM and Figlin RA: Sunitinib versus interferon alfa in metastatic renal-cell carcinoma. N Engl J Med 356(2): 115-124, 2007.

10 Kilonzo M1, Hislop J, Elders A, Fraser C, Bissett D, McClinton S, Mowatt G and Vale L: Pazopanib for the first-line treatment of patients with advanced and/or metastatic renal cell carcinoma: a NICE single technology appraisal. Pharmacoeconomics 31(1): 15-24, 2013.

11 Cowey CL and Rathmell WK: VHL gene mutations in renal cell carcinoma: Role as a biomarker of disease outcome and drug efficacy. Cur Oncol Rep 11(2): 94-101, 2009.

12 Hudes G, Carducci M, Tomczak P, Dutcher J, Figlin R, Kapoor A, Staroslawska E, Sosman J, McDermott D, Bodrogi I, Kovacevic Z, Lesovoy V, Schmidt-Wolf IG, Barbarash O, Gokmen E, O'Toole T, Lustgarten S, Moore L and Motzer RJ; Global ARCC Trial: Temsirolimus, interferon alfa, or both for advanced renal-cell carcinoma. N Engl J Med 356(22): 22712281, 2007.

13 Motzer RJ, Mazumdar M, Bacik J, Berg W, Amsterdam A and Ferrara J: Survival and prognostic stratification of 670 patients with advanced renal cell carcinoma. J Clin Oncol 17(8): 25302540, 1999.

14 Escudier B, Bellmunt J, Négrier S, Bajetta E, Melichar B, Bracarda S, Ravaud A, Golding S, Jethwa S, Sneller V: Phase III trial of bevacizumab plus interferon alfa-2a in patients with metastatic renal cell carcinoma (AVOREN): final analysis of overall survival. J Clin Oncol 28(13): 2144-2150, 2010.

15 Rini Brian I, Halabi Susan, Rosenberg Jonathan E, Stadler Walter M, Vaena Daniel A, Archer Laura et al: Phase III trial of bevacizumab plus interferon alfa versus interferon alfa monotherapy in patients with metastatic renal cell carcinoma: final results of CALGB 90206. J Clin Oncol 28(13): 2137-2143, 2010.

16 Rini BI, Halabi S, Rosenberg JE, Stadler WM, Vaena DA, Ou SS, Archer L, Atkins JN, Picus J, Czaykowski P, Dutcher J, Small EJ: Bevacizumab plus interferon alfa compared with interferon alfa monotherapy in patients with metastatic renal cell carcinoma: CALGB 90206. J Clin Oncol 26(33): 54225428, 2008.

17 Medical Research Council Renal Cancer Collaborators: Interferon$\alpha$ and survival in metastatic renal carcinoma: early results of a randomised controlled trial. Lancet 353(9146): 14-17, 1999.

18 Eichelberg C, Vervenne WL, De Santis M, Fischer von Weikersthal L, Goebell PJ, Lerchenmüller C, Zimmermann U, Bos MM, Freier W, Schirrmacher-Memmel S, Staehler M, Pahernik S, Los M, Schenck M, Flörcken A, van Arkel C, Hauswald K, Indorf M, Gottstein D and Michel MS: SWITCH: A randomised, sequential, open-label study to evaluate the efficacy and safety of sorafenib-sunitinib versus sunitinibsorafenib in the treatment of metastatic renal cell cancer. Eur Urol 68(5): 837-847, 2015.

19 ClinicalTrials.gov: Study in which therapy is either switched to nivolumab after 3 months of treatment or therapy is continued with a tyrosine kinase inhibitor in patients with metastatic renal cell carcinoma (RCC) and disease control (NIVOSWITCH). Available from: https://clinicaltrials.gov/ct2/show/NCT02959554 (Accessed on 04 December 2018).

20 Kaymakcalan MD, Xie W, Albiges L, North SA, Kollmannsberger CK, Smoragiewicz M, Kroeger N, Wells JC, Rha SY, Lee JL, McKay RR, Fay AP, De Velasco G, Heng DY and Choueiri TK: Risk factors and model for predicting toxicity-related treatment discontinuation in patients with metastatic renal cell carcinoma treated with vascular endothelial growth factor-targeted therapy: Results from the International Metastatic Renal Cell Carcinoma Database Consortium. Cancer 122(3): 411-419, 2016.

21 Mennitto A, Grassi P, Ratta R, Verzoni E, Prisciandaro M and Procopio G: Nivolumab in the treatment of advanced renal cell carcinoma: clinical trial evidence and experience. Ther Adv Urol 8(5): 319-326, 2016.

22 Lee $\mathrm{CH}$ and Motzer RJ: Immune checkpoint therapy in renal cell carcinoma. Cancer J 22(2): 92-95, 2016.

23 Escudier B, Tannir NM, McDermott DF, Frontera OA, Melichar B, Plimack ER, Barthelemy P, George S, Neiman V, Porta C, Choueiri TK, Powles T, Donskov F, Salman P, Kollmannsberger CK, Rini B, Mekan S, McHenry MB, Hammers HJ and Motzer RJ: LBA5CheckMate 214: Efficacy and safety of nivolumab + ipilimumab $(\mathrm{N}+\mathrm{I}) \mathrm{v}$ sunitinib $(\mathrm{S})$ for treatment-naïve advanced or metastatic renal cell carcinoma (mRCC), including IMDC risk and PD-L1 expression subgroups. Ann Oncol 28, 2017. doi: 10.1093/annonc/mdx440.029

24 Lee $\mathrm{CH}$, Makker V, Rasco DW, Taylor MH, Stepan DE, Shumaker RC, Schmidt EV, Guo M, Dutcus CE and Motzer RJ: Lenvatinib + pembrolizumab in patients with renal cell carcinoma: Updated results. Journal of Clinical Oncology 36(15_suppl): 4560-4560, 2018. 
25 Motzer RJ, Powles T, Atkins MB, Escudier B, McDermott DF, Suarez C, Bracarda S, Stadler WM, Donskov F, Lee JL, Hawkins RE, Ravaud A, Alekseev BY, Staehler MD, Uemura M, Donaldson F, Li S, Huseni MA, Schiff C, Rini BI: IMmotion151: A randomized phase III study of atezolizumab plus bevacizumab $v s$. sunitinib in untreated metastatic renal cell carcinoma (mRCC) oral abstract session C -renal cell carcinoma. ASCO 2018. J Clin Oncol 36(6_suppl): 578-578, 2018.

26 Atkins M, McDermott D, Powles T, Motzer R, Rini B, Fong L, Joseph R, Pal S, Sznol M, Hainsworth J, Stadler W, Hutson T, Ravaud A, Bracarda S, Suarez C, Choueiri T, Qiu J, Huseni M, Schiff C and Escudier B: IMmotion150: A phase II trial in untreated metastatic renal cell carcinoma (mRCC) patients (pts) of atezolizumab (atezo) and bevacizumab (bev) vs. and following atezo or sunitinib (sun). ASCO 2017. J Clin Oncol 35(15_suppl): 4505-4505, 2017.

27 McDermott DF, Atkins MB, Motzer RJ, Rini BI, Escudier BJ, Fong L, Joseph RW, Pal SK, Sznol M, Hainsworth JD, Stadler MW, Hutson TE, Ravaud A, Bracarda S, Suarez C, Choueiri TK, Choi YJ, Huseni MA, Fine GD and Powles T: A phase II study of atezolizumab (atezo) with or without bevacizumab (bev) versus sunitinib (sun) in untreated metastatic renal cell carcinoma (mRCC) patients (pts). J Clin Oncol 35(6_suppl): 431, 2017.

28 Unverzagt S, Moldenhauer I, Nothacker M, Roßmeißl D, Hadjinicolaou AV, Peinemann F, Greco F and Seliger B: Immunotherapy for metastatic renal cell carcinoma. Cochrane database Syst Rev 5: CD011673, 2017.
29 ESMO: eUpdate - Renal Cell Carcinoma Treatment Recommendations, 2017. Available from: https://www.esmo.org/ Guidelines/Genitourinary-Cancers/Renal-Cell-Carcinoma/ eUpdate-Renal-Cell-Carcinoma-Treatment-Recommendations

30 Leitlinienprogramm Onkologie: Leitlinie Diagnostik, Therapie und Nachsorge des Nierenzellkarzinoms Langversion 1.2, 2017. Available from: https://www.leitlinienprogramm-onkologie.de/ fileadmin/user_upload/Downloads/Leitlinien/Nierenzellkarzinom /LL_Nierenzell_Langversion_1.2.pdf.

31 DGHO Oncopedia: Leitlinie Nierenzellkarzinom (Hypernephrom), 2018. Available from: https://www.onkopedia.com/de/onkopedia/ guidelines/nierenzellkarzinom-hypernephrom/@@view/html/ index.html, Jan 2018.
Received September 28, 2018

Revised December 12, 2018

Accepted December 17, 2018 